\title{
Erratum to: Effect of calcination temperature on cobalt substituted cadmium ferrite nanoparticles
}

Ch. Venkata Reddy ${ }^{1}$ Chan Byon ${ }^{1} \cdot$ B. Narendra ${ }^{2} \cdot$ Bhaskar Dudem $^{2}$. Jaesool Shim ${ }^{1} \cdot$ Sang Jun Moon $^{3} \cdot$ S. V. Prabhakar Vattikuti ${ }^{1}$

Published online: 1 September 2015

(C) Springer Science+Business Media New York 2015

Erratum to: J Mater Sci: Mater Electron (2015) 26:5078-5084

DOI 10.1007/s10854-015-3031-2

This is to inform that the co-corresponding authors Sang Jun Moon and Jaesool Shim were missed out in the original publication during production process. Hence, this erratum is initiated to correct the error.

The online version of the original article can be found under doi:10.1007/s10854-015-3031-2.

$\triangle$ Ch. Venkata Reddy vijjivenki90@gmail.com

$\triangle$ Jaesool Shim

jshim@ynu.ac.kr

$\triangle$ Sang Jun Moon

nanobiomems@dgist.ac.kr

$凶$ S. V. Prabhakar Vattikuti vsvprabu@gmail.com

1 School of Mechanical Engineering, Yeungnam University, 214-1 Dae-dong, Gyeongsan-si, Gyeongsangbuk-do 712-749, Republic of Korea

2 Department of Physics, K L University, Vaddeswaram, AP 522 502, India

3 Cybernetics Laboratory, Daegu Gyeongbuk Institute of Science and Technology (DGIST), Daegu, Korea 